\title{
Multiwavelength Signatures of Shocks in Relativistic Jets
}

\author{
Markus Böttcher* \\ Centre for Space Research, North-West University, Potchefstroom, 2520, South Africa \\ E-mail: Markus.Bottcher@nwu.ac.za
}

\section{Matthew G. Baring}

Department of Physics and Astronomy, Rice University, Houston, TX 77005

E-mail: baring@rice.edu

\section{Errol J. Summerlin}

NASA Goddard Space Flight Center, Heliospheric Physics Laboratory, Code 671, Greenbelt, MD 20770, USA

E-mail: errol.summerlin@nasa.gov

\begin{abstract}
Mildly relativistic, oblique shocks are frequently invoked as possible sites of relativistic particle acceleration and production of strongly variable, polarized multi-wavelength emission from relativistic jet sources such as blazars, via diffusive shock acceleration (DSA). This paper summarizes recent results on a self-consistent coupling of DSA and radiation transfer simulations in blazar jets. We find that the observed spectral energy distributions (SEDs) of blazars strongly constrain the nature of the hydromagnetic turbulence responsible for pitch-angle scattering. Specifically, a strongly energy-dependent pitch-angle mean free path is required. In the case of lowfrequency-peaked blazars, we find that the scaling of the pitch-angle-scattering mean-free-path, $\lambda_{\text {pas }}$ sometimes needs to be as strong as $\lambda_{\text {pas }} \propto p^{3}$, where $p$ is particle momentum. As our model self-consistently reproduces the relative normalization of thermal vs. non-thermal particles heated/accelerated at the shock, Compton scattering of an external radiation field by the thermal population produces a bulk Compton feature in the SED. This feature is appropriate to model the prominent soft X-ray excess in the SED of the BL Lac object AO 0235+164, which places additional constraints on the level of hydromagnetic turbulence. A prediction of this interpretation is that the soft X-ray emission in this soft excess is expected to be highly polarized.
\end{abstract}

4th Annual Conference on High Energy Astrophysics in Southern Africa

25-27 August, 2016

Cape Town, South Africa

${ }^{*}$ Speaker. 


\section{Introduction}

Relativistic, oblique shocks have long been considered one of the leading contenders for the sites of relativistic particle acceleration, resulting in the observed rapidly variable, often highly polarized multi-wavelength emission from relativistic jet sources, such as blazars and gamma-ray bursts. The dominant mechanism of particle acceleration at such shocks is referred to as diffusive shock acceleration (DSA). Particle acceleration results from repeated shock crossings of particles gyrating along large-scale ordered magnetic fields. The reversal of particle momenta along magnetic field lines is facilitated by diffusive pitch-angle scatterings. Several theoretical studies of particle acceleration at relativistic shocks (e.g., $[12,8,9,19])$ have shown that this process can result in a wide variety of spectral indices, $q \geq 1$, where $n(p) \propto p^{-q}$ is the particle distribution. Such studies of the particle acceleration mechanism, however, usually do not consider the resulting radiative signatures in a self-consistent manner.

On the other hand, models focusing on the multi-zone radiative transfer problem have reached an increasing level of sophistication (e.g., $[13,18,16,14,17,10,4,11,6,7])$ and have resulted in successful fits to multi-wavelength spectral energy distributions (SEDs) and light curves of individual flares in blazars. In all cited works, however, the details of particle acceleration are not addressed, but shock acceleration is assumed to result in the injection of purely non-thermal relativistic particles, typically with a truncated power-law distribution in energy. In a recent work, we [3], we have coupled the Monte-Carlo simulations of DSA of Summerlin \& Baring [19] with radiative transfer routines of Böttcher et al. [5]. For the first time, this provides a consistent description of the DSA process in mildly relativistic, oblique shocks in blazar jets and its radiative signatures. The results of that study are summarized in this conference contribution, focusing on implications for the nature of hydromagnetic turbulence facilitating pitch-angle scattering.

\section{Diffusive Shock Acceleration in Relativistic Jets}

Results of detailed Monte-Carlo (MC) simulations of DSA at relativistic, oblique shocks have recently been published by Summerlin \& Baring [19]. In the DSA scenario, the Fermi-I acceleration process is facilitated by pitch-angle scattering, causing the guiding centers of particles spiraling along magnetic field lines to reverse their direction of motion with respect to the shock in a stochastic manner. The microphysical details of pitch-angle scattering depend on the (unknown) details of the hydromagnetic turbulence created by the passage of the shock. As a self-consistent hydrodynamical treatment of the generation and cascading of turbulence along with $\mathrm{MC}$ simulations of DSA is beyond current computational capabilities, [19] parameterized pitch-angle scattering through the mean-free path $\lambda_{\text {pas }}$ as an energy-dependent multiple $\eta(p)$ of the particle's gyro radius, $r_{g}=p c /(q B)$, where $p$ is the particle's momentum, such that $\lambda_{\text {pas }}=\eta(p) r_{g}$. The energy dependence of the mean-free-path parameter $\eta$ is defined as a power-law in the particle's momentum, $\eta(p)=\eta_{1} p^{\alpha-1}$, so that $\lambda_{\text {pas }} \propto p^{\alpha}$ and $\eta_{1}$ describes the mean free path in the non-relativistic limit, $\gamma \rightarrow 1$. The work of [19] focused on the special case $\alpha=1$. Here we generalize this study to consider different values of $\alpha$.

The MC simulations of [19] illustrate that the DSA process leads to a non-thermal powerlaw tail of relativistic particles which have been accelerated out of the remaining thermal pool. 
A high-energy cut-off $\left(\gamma_{\max }\right)$ of the non-thermal particle spectra results from the balance of the acceleration time scale $t_{\mathrm{acc}}\left(\gamma_{\max }\right)=\eta\left(\gamma_{\max }\right) t_{\mathrm{gyr}}\left(\gamma_{\max }\right)$ with the radiative energy loss time scale. If synchrotron losses dominate, $\gamma_{\max } \propto B^{-1 / 2}$. This will lead to a synchrotron peak energy $E_{\mathrm{sy}} \sim$ $240 \delta \eta^{-1}\left(\gamma_{\max }\right) \mathrm{MeV}$, where $\delta$ is the Doppler factor associated with the bulk motion of the particle acceleration region. Notably, this synchrotron peak energy is independent of the magnetic field $B$, as $E_{\mathrm{sy}} \propto B \gamma_{\max }^{2}$. Blazars typically show synchrotron peaks in the IR to soft X-rays. In order to reproduce these, the pitch-angle-scattering mean-free-path parameter $\eta\left(\gamma_{\max }\right)$ has to assume values of $\sim 10^{4}-10^{8}$, depending on the location of the synchrotron peak. However, [19] have shown that $\eta_{1}$ must be significantly smaller than this value in order to obtain efficient injection of particles out of the thermal pool into the non-thermal acceleration process. From these arguments we can infer that $\eta(\gamma)$ must be strongly energy dependent. The inferences of such a strong energy dependence will be discussed in more detail below.

Radiative losses will steepen the power-law non-thermal particle spectrum by $\Delta q=1$ for particles at energies below $\gamma_{\max }$. The break from $n(p) \propto p^{-q}$ to $n(p) \propto p^{-(q+1)}$ occurs at a break energy $\gamma_{b}$ where the radiative cooling time scale equals the dynamical (particle escape) time scale. The radiative cooling time scale, required for the evaluation of both $\gamma_{\max }$ and $\gamma_{b}$ is calculated selfconsistently with the radiation transfer problem described in the next Section.

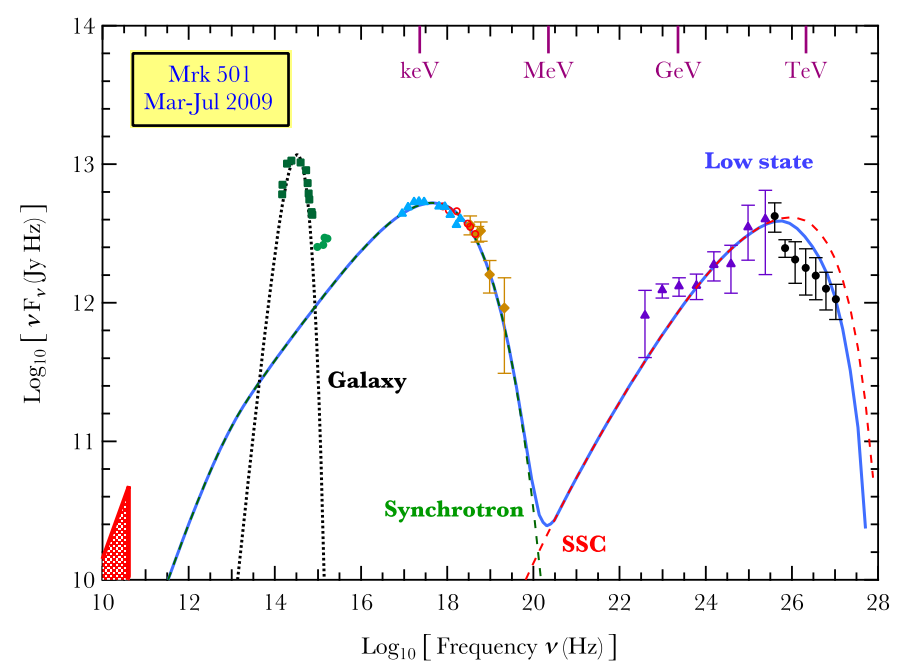

Figure 1: Fit to the SED of the HBL Mrk 501 (data from [1]), with $\eta_{1}=100$ and $\alpha=1.5$. From [3].

\section{Radiation Modeling}

The novel aspect of the work of [3] consisted of the self-consistent coupling of the MC simulations of DSA of [19], to radiative transport simulations, using the routines of [5]. Synchrotron emission in a tangled magnetic field, synchrotron self-Compton scattering and Compton scattering of external radiation fields are taken into account self-consistently both in the calculation of radiative energy loss rates of relativistic electrons, and of the radiative output. 
We have used our coupled DSA MC + radiation-transfer simulations to model the SEDs of three blazars: the high-frequency-peaked BL Lac object (HBL) Mrk 501 and the two lowfrequency-peaked BL Lac objects (LBLs) BL Lacertae and AO 0235+164. As detailed in [3], some of the model parameters can be independently constrained from observables such as the mimum variability time scale, superluminal motion measurements, and the synchrotron and Compton peak frequencies.

Figure 1 shows the resulting fit to the SED of the HBL Mrk 501 (data from [1]). As typical for HBLs, its synchrotron peak is usually located at soft X-ray energies. We found that a choice of $\eta_{1}=$ 100 and $\alpha=1.5$, i.e., $\eta(\gamma)=100 \gamma^{0.5}$ resulted both in efficient relativistic particle acceleration out of the thermal pool and a synchrotron spectrum matching well the observed SED of Mrk 501. Other parameters used for our fit are typical of leptonic synchrotron self-Compton fits to HBLs in general and Mrk 501 in particular. For details see [3]. We note that not all details of the $\gamma$-ray spectrum are well reproduced by our model. This could certainly be improved by introducing additional free parameters (e.g., additional electron populations and radiation components). However, we refrain from such fine-tuning as the goal of this work is to demonstrate that the self-consistent coupling of DSA and radiation transfer, with a minimal number of free parameters, provides a satisfactory representation of the observed SED of Mrk 501.

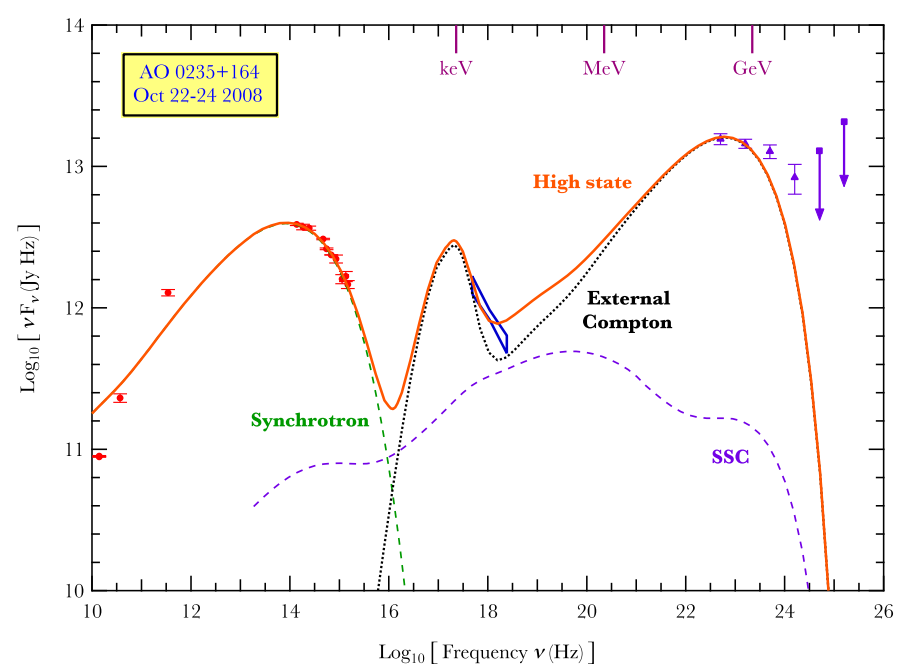

Figure 2: Fit to the SED of the LBL AO $0235+164$ (data from [2]), with $\eta_{1}=225$ and $\alpha=3$. Note that the soft X-ray excess is reproduced by the bulk Compton emission of the thermal electron population. From [3].

In Figure 2 we show an example of an LBL, namely AO 0235+164 (data from [2]). In this case, the synchrotron peak is located in the IR, requiring a much more extreme energy dependence of $\eta$. We have achieved this with the choice of $\eta_{1}=225$ and $\alpha=3$, i.e., $\eta(\gamma)=225 \gamma^{2}$. The same scaling of $\eta$ with particle momentum was required for BL Lacertae, which is not shown here. The flare-state SED of AO 0235+164 exhibits a pronounced soft X-ray excess indicated by the steep Swift-XRT $2-10 \mathrm{keV}$ spectrum. This excess clearly indicates a separate radiation component beyond the standard, non-thermal synchrotron, SSC, and external-Compton components (shown by the dotted, dashed, and dot-dashed curves in Figure 2, respectively). Our self-consistent coupling of 
thermal particle heating and non-thermal particle acceleration via DSA allows us to reproduce this component with the bulk Compton mechanism [15]. In this process, the thermal electron population Compton up-scatters the external radiation field (dominated by IR emission from a dusty torus) by a factor $\sim \Gamma^{2}$, where $\Gamma$ is the bulk Lorentz factor of the emission region. This tightly constrains the thermal-to-non-thermal normalization of the electron population and thereby the parameter $\eta_{1}$. If the soft X-ray excess is indeed due to Compton scattering by thermal electrons, this strongly anisotropic Compton scattering process is expected to induce significant linear polarization due to the polarization-dependence of the Compton cross section. This polarization effect disappears in the case of Compton scattering by relativistic electrons, as the angular characteristic of the target photon distribution becomes dominated by the relativistic aberration into the electron rest frame, washing out any macroscopic anisotropy. Thus, the degree of polarization is expected to decrease to almost zero towards hard X-rays (for detailed calculations of these X-ray polarization features, see Garrigoux et al. 2017, in preparation).

As for Mrk 501, obviously not all SED points are well represented by our model. To preserve the self-consistency of our approach with a minimal number of free parameters, we again refrain from introducing additional processes (and free parameters) which may be able to improve the fit.

\section{Turbulence Characteristics}

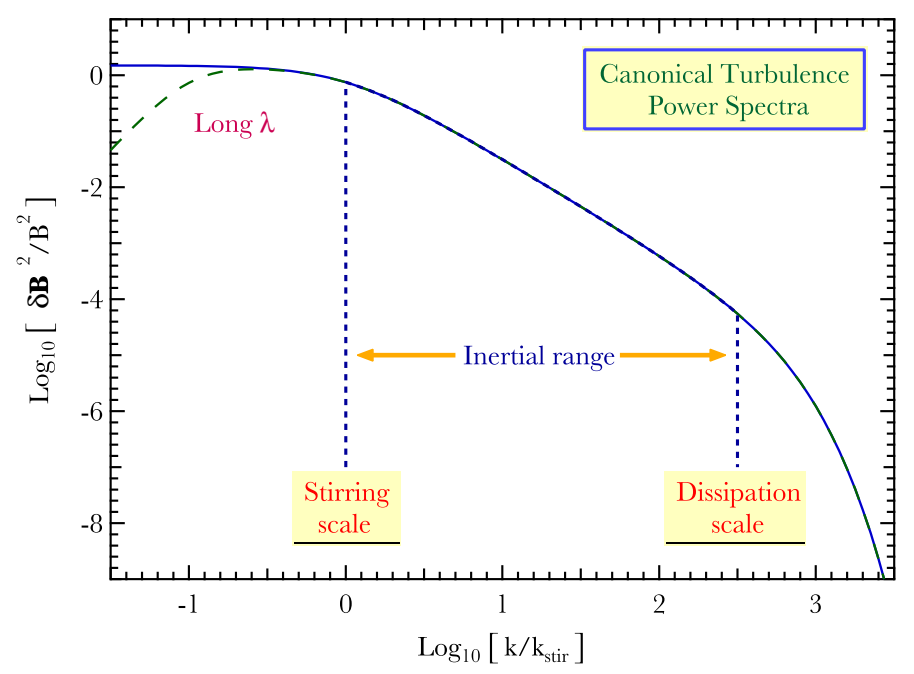

Figure 3: Sketch of a magneto-hydrodynamic turbulence spectrum plausibly expected in the post-shock region. Waves are excited at the longest wavelengths corresponding to the stirring scale, and cascade down to smaller scales, down to the dissipation scale. From [3].

All of our SED fits that we have produced with our coupled DSA + radiation transfer simulations implied a strong energy dependence of the pitch-angle mean free path. This provides interesting insights into the characteristics of hydromagnetic turbulence in the blazar jet. Pitch-angle scattering is most efficient in the gyro-resonant regime, in which particles interact with plasma 
waves of the same (co-moving) frequency as their own gyro-frequency. Gyro-resonant particlewave interactions therefore occur with waves of wave number $k_{\|}$in the propagation direction along the guiding magnetic field, given by $k_{\|}=\omega_{B} /(\gamma \nu|\mu|)$ where $\omega_{B}$ is the particle's gyro-frequency, $v$ its velocity, and $\mu$ the cosine of its pitch angle. To first order, this implies that the resonant wave length for a particle with energy $\gamma$ scales as $\lambda_{\text {res }} \propto \gamma$. Consequently, the highest-energy particles will interact primarily with the longest wavelength plasma waves (smallest $k_{\|}$). Two effects are expected to contribute to the suppression of such gyro-resonant interactions for very long wavelengths: (1) For wavelengths approaching (and exceeding) the "stirring scale" on which plasma turbulence is induced, the energy density contained in such waves is rapidly decreasing with increasing wavelengths (see Figure 3); (2) the highest energy particles will probe large volumes behind the shock front, where the level of turbulence is expected to decrease with distance from the shock. These effects provide a natural explanation of the strong energy dependence of the pitch-angle mean free path which we find in our analyses. The internal consistency of this picture provides support for DSA as the dominant particle acceleration mechanism in blazar jets.

\section{ACKNOWLEDGMENTS}

The work of M. Böttcher is supported through the South African Research Chairs Initiative (grant no. 64789) of the Department of Science and Technology and the National Research Foundation ${ }^{1}$ of South Africa. This work was further supported by NASA through Astrophysics Theory Program grant NNX10AC79G and by the Department of Energy under grant DE-SC0001481.

\section{References}

[1] Abdo, A. A., et al., 2011, ApJ, 727, 129

[2] Ackermann, M., et al., 2012, ApJ, 751, 159

[3] Baring, M. G., Böttcher, M., \& Summerlin, E. J., 2017, MNRAS, 464, 4875

[4] Böttcher, M., \& Dermer, C. D., 2010, ApJ, 711, 445

[5] Böttcher, M., Reimer, A., Sweeney, K., \& Prakash, A., 2013, ApJ, 768, 54

[6] Chen, X., Fossati, G., Liang, E. P., \& Böttcher, M., 2011, MNRAS, 416, 2368

[7] Chen, X., Fossati, G., Böttcher, M., \& Liang, E. P., 2012, MNRAS, 424, 789

[8] Ellison, D. C., Reynolds, S. P., \& Jones, F. C., 1990, ApJ, 360, 702

[9] Ellison, D. C., \& Double, G. P., 2004, Astropart. Phys., 22, 323

[10] Graff, P. B., Georganopoulos, M., Perlman, E. S., \& Kazanas, D., 2008, ApJ, 689, 68

[11] Joshi, M., \& Böttcher, M., 2011, ApJ, 727, 21

[12] Kirk, J. G., \& Heavens, A. F., 1989, MNRAS, 239, 995

[13] Marscher, A. P., \& Gear, W. K., 1985, ApJ, 298, 114

\footnotetext{
${ }^{1}$ Any opinion, finding and conclusion or recommendation expressed in this material is that of the authors and the NRF does not accept any liability in this regard.
} 
[14] Mimica, P., Aloy, M. A., Müller, E., \& Brinkmann, W., 2004, A\&A, 418, 947

[15] Sikora, M., Madejski, G., Moderski, R., \& Poutanen, J., 1997, ApJ, 484, 108

[16] Sokolov, A., Marscher, A. P., \& McHardy, I. M., 2004, ApJ, 613, 725

[17] Sokolov, A., \& Marscher, A. P., 2005, ApJ, 625, 52

[18] Spada, M., Ghisellini, G., Lazzati, D., \& Celotti, A., 2001, MNRAS, 325, 1559

[19] Summerlin, E. J., \& Baring, M. G., 2012, ApJ, 745, 63 